\title{
Uso de contracepção e desigualdades do planejamento reprodutivo das mulheres brasileiras
}

\author{
Contraception use and family planning inequalities \\ among Brazilian women
}

Raquel Elias da Trindade (https://orcid.org/0000-0002-6854-1255) ${ }^{1}$

Bárbara Barrozo Siqueira (https://orcid.org/0000-0003-1813-128X) ${ }^{2}$

Thayane Fraga de Paula (https://orcid.org/0000-0003-1428-3104) ${ }^{2}$

Mariana Santos Felisbino-Mendes (https://orcid.org/0000-0001-5321-5708) ${ }^{2}$

${ }^{1}$ Curso de Graduação em Enfermagem, Departamento de Enfermagem MaternoInfantil e Saúde Pública. Escola de Enfermagem, Universidade Federal de Minas Gerais. Av. Prof. Alfredo Balena 190, Santa Efigênia. 30130-100 Belo Horizonte MG Brasil. marianafelisbino@ yahoo.com.br

${ }^{2}$ Programa de PósGraduação em Enfermagem, Departamento de Enfermagem MaternoInfantil e Saúde Pública, Escola de Enfermagem, Universidade Federal de Minas Gerais. Belo Horizonte MG Brasil.

\begin{abstract}
Background: Brazil has shown a considerable decline in fertility rates in recent decades. However, sociodemographic differences still have a direct impact on access to family planning in the country. Objective: To estimate the prevalence of contraceptive use according to sociodemographic variables among Brazilian women in reproductive age. Methods: A crosssectional study conducted with 17,809 women who have responded to the National Health Survey. We estimated the prevalence as well as the 95\% confidence intervals and we used Pearson's chi-square test at a significance level of 5\% to analyze differences between groups. Results: More than $80 \%$ of the women reported to use some contraception method, the most used method was oral contraceptive (34.2\%), followed by surgical (25.9\%) and condoms (14.5\%). Black/Brown, northerly, and low-educated women are more frequently sterilized, while white women, with higher schooling and those living in the south and southeast are the ones who use oral contraception and double protection the most. Conclusion: Despite the observed improvements, there was no decrease in the prevalence for not using any CM and there are inequalities in access to contraception in the country.

Key words Family planning, Public health, Women's health, Contraception, Health status disparities, Nursing
\end{abstract}

Resumo O Brasil apresentou elevada queda de fecundidade nas últimas décadas. No entanto, diferenças sociodemográficas ainda impactam diretamente no acesso ao planejamento reprodutivo no país. O objetivo deste artigo é estimar a prevalência do uso de métodos contraceptivos (MC) de acordo com variáveis sociodemográficas entre mulheres brasileiras em idade reprodutiva. Tratase de um estudo transversal que utilizou dados secundários de 17.809 mulheres que responderam à Pesquisa Nacional de Saúde. Estimou-se a prevalência com intervalos de 95\% de confiança e utilizou-se o teste qui-quadrado de Pearson com nível de significância de 5\%. Mais de 80\% das mulheres relataram utilizar algum $M C$, sendo o contraceptivo oral o mais utilizado (34,2\%), seguido dos cirúrgicos (25,9\%) e das camisinhas (14,5\%). As mulheres pretas/pardas, nortistas e com baixa escolaridade são mais esterilizadas, enquanto as brancas, com maior escolaridade e das regiões Sul e Sudeste são as que mais utilizam contraceptivo oral e dupla proteção. Apesar das melhorias observadas não houve diminuição da prevalência do não uso de MC e ainda existem desigualdades de acesso à contracepção no país.

Palavras-chave Planejamento familiar, Saúde pública, Saúde da mulher, Anticoncepção, Disparidades nos níveis de saúde, Enfermagem 


\section{Introdução}

Nas últimas décadas, o Brasil apresentou alta queda em sua fecundidade, assim como vários países da América Latina. Dados do Instituto Brasileiro de Geografia e Estatística (IBGE) demonstram que a taxa de fecundidade de 6,3 filhos, em 1960, diminuiu para 1,7 filho por mulher em $2015^{1}$. Contudo, essa redução aconteceu de diferentes formas entre as classes sociais. Primeiramente, ocorreu entre as mulheres que tinham maior escolaridade e que habitavam as zonas urbanas. Essa desigualdade pode ser observada no Brasil a partir dos dados do Censo de 2010, que demonstraram taxa de fecundidade das regiões menos desenvolvidas do país, Norte e Nordeste, de respectivamente 2,4 e 2,0 filhos, enquanto nas regiões mais desenvolvidas, Sul e Sudeste, eram de 1,8 e 1,7 filho por mulher².

Apesar dessa redução, mais da metade das gestações que ocorrem no Brasil não são planejadas, segundo a pesquisa nacional Nascer no Brasil ${ }^{3}$. Além disso, as mulheres que planejam suas gestações são, na maioria, brancas, com maior nível de instrução escolar, com mais de 35 anos de idade e se encontram em um relacionamento estável ${ }^{4}$.

O indicador de demanda por planejamento familiar satisfeita (DPFS) avalia mulheres que usam algum método contraceptivo (MC) entre as que necessitam utilizar, ou seja, mulheres fecundas que não querem engravidar nos próximos dois anos ou não têm certeza se querem ${ }^{5}$. Pesquisa recente demonstrou que, no Brasil, a taxa de DPFS entre mulheres casadas ou em união estável foi a segunda maior da América Latina $(94,7 \%)^{6}$. No entanto, trata-se de resultado que se limita às mulheres casadas, não se constituindo nem a metade da população feminina no Brasil em idade fértil ${ }^{7}$.

Estudo desenvolvido em 2018 com dados de 77 países evidenciou, a partir da análise de DPFS, que os níveis mais baixos desse indicador ocorrem entre as mulheres mais jovens, pobres e que habitam a zona rural. Esse resultado mostra que mulheres com critérios socioeconômicos que as colocam em posição de vulnerabilidade estão mais propensas a não usar MC e, consequentemente, a ter uma gestação não planejada. Além disso, evidencia a necessidade de se incluir todas as mulheres nas pesquisas de planejamento reprodutivo (PR). Nessa pesquisa, o Brasil não foi considerado $^{8}$, provavelmente devido à limitação dos dados dos inquéritos nacionais mais recentes sobre o tema.

Além do uso, observa-se que o ranking dos MC varia de acordo com condições socioeconômicas e aspectos culturais da população. No México, por exemplo, entre os mais utilizados estão os métodos cirúrgicos na população feminina e o dispositivo intrauterino (DIU) ${ }^{9}$. Nos Emirados Árabes, a camisinha lidera o ranking, seguida do DIU e do coito interrompido. Já a vasectomia não entra no ranking desse país, o que reforça a desigualdade de gênero ${ }^{10}$.

Cabe ainda ressaltar que a Pesquisa Nacional de Demografia e Saúde da Criança e da Mulher (PNDS) de 2006 foi o último inquérito nacional que tratou o PR como um objetivo estruturante da pesquisa. No entanto, em suas publicações, considerou apenas as mulheres com companheiro para investigar o uso dos métodos de contracepção e as condições sociodemográficas das mulheres ${ }^{11}$, desconsiderando mulheres sem companheiros que também se beneficiariam da contracepção.

As ações de PR são importantes intervenções de saúde, pois o uso dos MC e, consequentemente, a redução do tamanho das famílias acarretam redução da morbimortalidade ${ }^{12}$. No Brasil, por exemplo, a mortalidade materna por complicações do aborto se configura como a quinta causa de óbitos no país ${ }^{13}$. Dados da Pesquisa Nacional do Aborto, de 2016, evidenciaram que 13\% das mulheres entrevistadas tiveram um abortamento provocado por não desejar a gestação ${ }^{14}$.

Identificar os indicadores de cobertura, ou seja, a proporção de pessoas que recebem o serviço que desejam ${ }^{12}$, torna-se fundamental para formular políticas públicas de saúde que visam o bem-estar materno e infantil ${ }^{10}$. Nesse caso, estamos falando de todas as mulheres em idade fértil, independentemente do estado civil.

Acredita-se que analisar a prevalência do uso e o ranking dos $\mathrm{MC}$, segundo as características sociodemográficas, como proposto neste estudo, possibilita investigar diferenças importantes no PR das mulheres brasileiras. Portanto, os objetivos foram estimar a prevalência do uso dos MC, enumerar os motivos do não uso, mostrar quais são os métodos mais utilizados e a prevalência do uso de MC, de acordo com as variáveis sociodemográficas entre as mulheres brasileiras em idade reprodutiva. 


\section{Materiais e métodos}

\section{Tipo de estudo e fonte de dados}

Trata-se de um estudo transversal de base populacional que utilizou dados secundários da Pesquisa Nacional de Saúde (PNS) relativos ao "Módulo R", que contém informações referentes a saúde da mulher, planejamento reprodutivo e contracepção, esses publicamente disponíveis no site https://www.ibge.gov.br/estatisticas/sociais/saude/9160-pesquisa-nacional-de-saude. html?=\&t=downloads.

\section{Pesquisa Nacional de Saúde de 2013 (PNS-2013)}

A PNS é a pesquisa de saúde mais atual e completa, realizada nos domicílios de todo território nacional, em parceria com o IBGE, a Fundação Oswaldo Cruz (Fiocruz) e o Ministério da Saúde (MS). Seu principal objetivo foi produzir dados sobre as questões de saúde dos brasileiros, como estilo de vida, estado de saúde, acesso aos serviços, promoção à saúde, continuidade do cuidado, entre outras ${ }^{15}$.

A coleta de dados ocorreu entre 2012 e 2013 e o plano amostral ocorreu em três estágios: o primeiro se referiu às unidades primárias de amostragem (UPA); o segundo, ao sorteio dos domicílios; e o terceiro, à escolha de um indivíduo acima de 18 anos. Em cada etapa, o sorteio foi por amostragem aleatória simples. A pesquisa utilizou três questionários, sendo um sobre as características da moradia, outro acerca dos moradores da mesma e o terceiro com informações do morador escolhido ${ }^{16}$. As UPA consideradas fazem parte de uma subamostra da amostra mestra do IBGE e a seleção dos municípios foi executada de acordo com o Cadastro Nacional de Endereço para Fins Estatísticos (CNEFE) mais recente no ano da pesquisa ${ }^{17}$.

As perguntas do inquérito foram separadas em módulos de "A" a " $X$ ". Utilizamos as questões do módulo "R", referente à saúde da mulher. Nele, as mulheres foram indagadas acerca de temas como a realização do exame preventivo do câncer de colo do útero, mamografia, menstruação e menopausa, história reprodutiva e planejamento familiar e contracepção. Também foram utilizadas as perguntas dos módulos "A" (informações do domicílio), "C" (características gerais dos moradores), "D” (características de educação das pessoas de cinco anos ou mais de idade) e "I" (co- bertura de plano de saúde) para obter informações sobre as características sociodemográficas ${ }^{15}$.

\section{População}

Foram entrevistados 60.202 indivíduos com mais de 18 anos. Desses, 34.282 eram mulheres, sendo que 22.621 estavam em idade reprodutiva (18 a 49 anos). Foram excluídas mulheres que relataram que não menstruam $(\mathrm{n}=998)$ e que não tiveram relações sexuais nos últimos 12 meses $(\mathrm{n}=3.155)$, totalizando 17.809 mulheres estudadas (Figura 1).

Portanto, os critérios de exclusão deste estudo se baseiam no fato de que essas mulheres não estavam expostas à possibilidade de engravidar. Ou seja, as que fizeram histerectomia, estão na menopausa ou nunca menstruaram e aquelas que não possuem vida sexual ativa ${ }^{8}$.

O MS define como mulheres férteis aquelas que possuem entre 10 e $49 \operatorname{anos}^{18}$. No entanto, a PNS foi realizada apenas com maiores de 18 anos e possui dados de mulheres até 49 anos, por isso definimos a faixa etária de mulheres em idade reprodutiva como aquelas possuem de 18 a 49 anos.

\section{Variáveis dependentes}

As variáveis utilizadas foram o uso e não uso de método contraceptivo. Para o uso, os MC foram separados em sete categorias. A primeira foi composta pelos métodos definitivos, ou seja, as cirurgias de esterilização (laqueadura/ligadura de trompas e vasectomia). Depois, os métodos hormonais foram segregados entre a pílula anticoncepcional e os outros métodos hormonais (contraceptivo injetável e implante intradérmi$\mathrm{co})^{6,8,10}$. O quarto e quinto grupos se originaram dos métodos que, além de contraceptivos, também previnem infecções sexualmente transmissíveis (IST): as camisinhas (feminina e masculina) e a dupla proteção (combinação dos métodos hormonais ou dispositivo intrauterino, diafragma e creme/óvulo com alguma das camisinhas) ${ }^{19}$. A sexta categoria constitui-se do dispositivo intrauterino (DIU). Por fim, foi criado um grande grupo denominado como outros métodos contraceptivos, composto pelo método tradicional conhecido como "tabelinha", métodos com pouca adesão (creme/óvulo e diafragma), método de emergência (pílula do dia seguinte), outros citados pela mulher que não estavam como opção no questionário e outras combinações que não configuram dupla proteção ${ }^{6,8,10}$. 


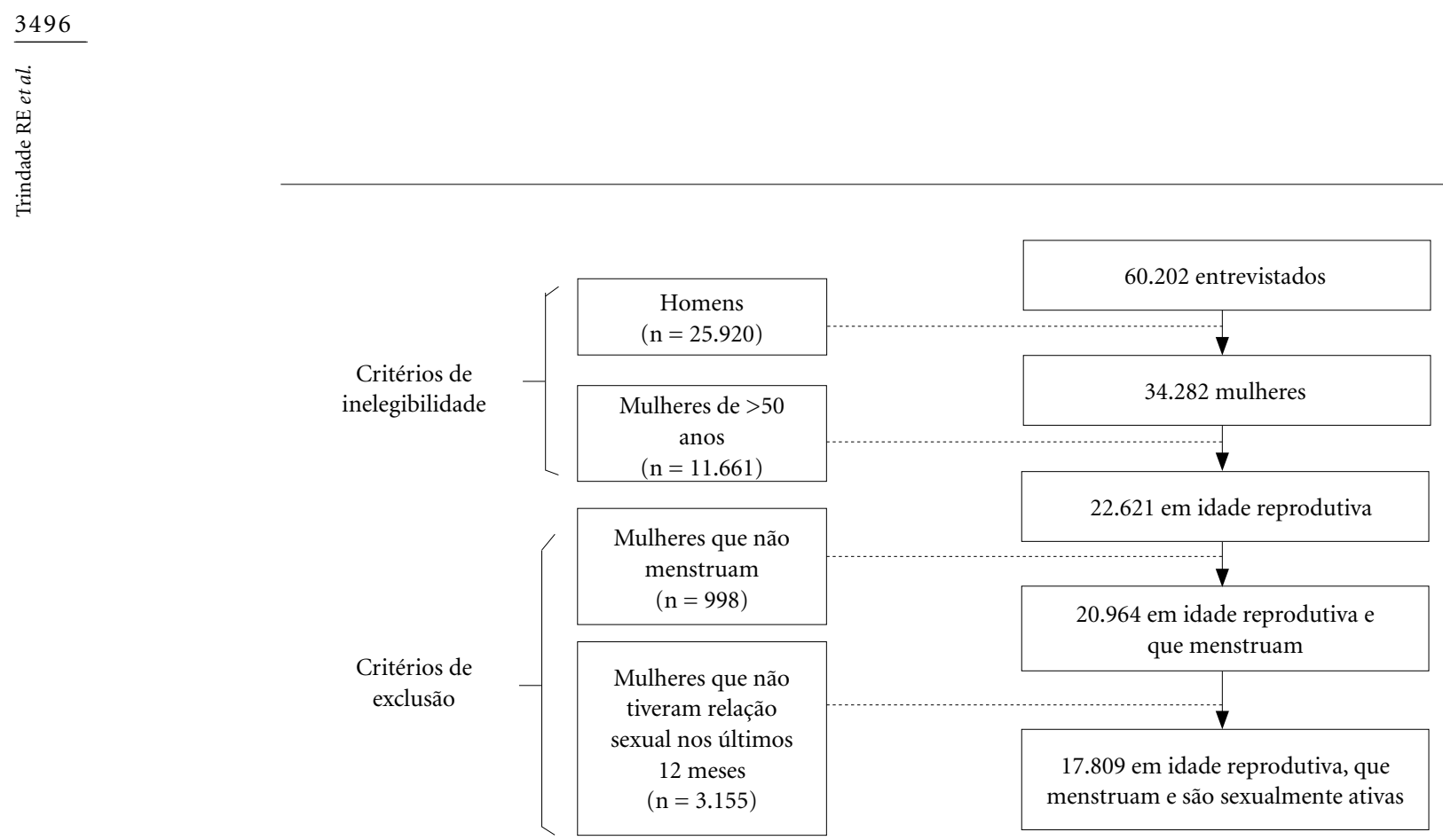

Figura 1. Fluxograma da população estudada.

Fonte: Elaborado pelos autores.

\section{Variáveis independentes}

As variáveis independentes utilizadas foram: região do Brasil (Norte, Nordeste, Centro-Oeste, Sudeste e Sul), zona de moradia (rural ou urbana), estado civil (com companheiro ou sem companheiro), cor da pele/raça (branco, preto/pardo e amarela/indígena), idade ( 18 a 24 anos, 25 a 34 anos e 35 a 49 anos), escolaridade ( 0 a 8 anos de estudo, 9 a 11 e 12 ou mais) e posse de plano de saúde (sim ou não).

\section{Análise dos dados}

Inicialmente, estimou-se a prevalência de uso de MC na população de mulheres brasileiras em idade reprodutiva. Em seguida, foram estimadas as prevalências dos motivos de não uso dos métodos, e depois foi definido o ranking dos métodos de contracepção em ordem decrescente de uso. Ao final, foram analisadas as prevalências do uso em relação às variáveis sociodemográficas. Para cada prevalência, estimou-se intervalos de $95 \%$ de confiança (IC 95\%) e aplicou-se teste qui-quadrado de Pearson ( $\mathrm{p}<0,05)$.

Por meio do programa estatístico Stata, versão 15.0, e os comandos do módulo survey, consideramos os pesos, UPA e conglomerados, mantendo a representatividade das amostras.

\section{Aspectos éticos}

A PNS foi aprovada em 2013 pelo Comitê Nacional de Ética em Pesquisa para Seres Humanos do Ministério da Saúde, sob o parecer 749/2006. Foi preservado o sigilo de todos os participantes e todos estiveram de acordo com a pesquisa por meio da assinatura do termo de consentimento livre e esclarecido. Por se tratar de um estudo que utiliza dados secundários, não identificados e com informações de acesso público, a Resolução no 466, de 12 de dezembro de 2012, dispensa a aprovação prévia em comitê de ética.

\section{Resultados}

Mais de $80 \%$ das mulheres relataram utilizar algum método contraceptivo. Das $17.809 \mathrm{mu}$ lheres de 18 a 49 anos que menstruam e são sexualmente ativas, $17,6 \%(n=3.181)$ afirmaram não utilizar nenhum MC. O principal motivo apresentado para o não uso de contraceptivos foi vontade própria ou não se importar de engravidar (37,3\%). Destaca-se, ainda, o fato de que $3,7 \%$ das brasileiras não evitam a gravidez, pois não sabem como evitar ou aonde ir ou a quem procurar para lhe dar orientações (Tabela 1). 
Tabela 1. Motivos para o não uso de contracepção entre as mulheres brasileiras em idade reprodutiva (18 a 49 anos).

\begin{tabular}{lrrr}
\hline \multicolumn{1}{c}{ Motivo } & \multicolumn{1}{c}{$\mathbf{n}^{\star}$} & \% $^{\star *}$ & IC 95\% \\
\hline Quer engravidar ou não se importa de engravidar & 1.251 & 37,3 & $34,5-40,2$ \\
Outros motivos $^{\star * \star *}$ & 855 & 30,6 & $27,7-33,7$ \\
Está grávida & 602 & 16,3 & $14,3-18,6$ \\
Não tem relações sexuais com homens & 233 & 8,0 & $6,4-10,1$ \\
Motivos religiosos & 117 & 4,1 & $3,0-5,6$ \\
Não sabe como evitar & 73 & 2,6 & $1,7-3,9$ \\
Não sabe aonde ir ou a quem procurar para lhe dar & 50 & 1,1 & $1,0-1,7$ \\
orientações & & &
\end{tabular}

* Número amostral; ${ }^{* *}$ prevalência populacional; ${ }^{* * *}$ intervalo de $95 \%$ de confiança; ${ }^{* * *}$ outros motivos especificados pela mulher não disponíveis no questionário.

Fonte: Elaborado pelos autores.

Tabela 2. Ranking dos métodos contraceptivos utilizados por brasileiras em idade reprodutiva (18 a 49 anos).

\begin{tabular}{|c|c|c|c|}
\hline Método Contraceptivo & $\mathbf{n}^{*}$ & $\%^{* *}$ & IC $95 \% \%^{* * *}$ \\
\hline Contraceptivo oral & 4.235 & 34,2 & $32,9-35,6$ \\
\hline Cirúrgicos $^{1}$ & 4.084 & 25,9 & $24,6-27,2$ \\
\hline Camisinhas $^{2}$ & 2.579 & 14,5 & $13,5-15,5$ \\
\hline Dupla proteção ${ }^{3}$ & 1.455 & 9,8 & $8,9-10,7$ \\
\hline Outros métodos ${ }^{4}$ & 1.088 & 7,8 & $7,1-8,6$ \\
\hline Outros hormonais ${ }^{5}$ & 924 & 6,0 & $5,4-6,7$ \\
\hline $\mathrm{DIU}^{6}$ & 263 & 1,8 & $1,5-2,3$ \\
\hline Total & 14.628 & 100 & - \\
\hline
\end{tabular}

${ }^{*}$ Número amostral; ${ }^{* *}$ prevalência populacional; ${ }^{* * *}$ intervalo de $95 \%$ de confiança; ${ }^{1}$ vasectomia; ${ }^{2}$ camisinhas feminina e masculina; ${ }^{3}$ dupla proteção - combinação entre camisinhas e métodos hormonais, diafragma, DIU ou óvulo/creme; ${ }^{4}$ outros métodos - tabela, óvulo/creme, diafragma, PDS e outros métodos e combinações que não configuram dupla proteção; ${ }^{5}$ outros hormonais - injetáveis e implante intradérmico; ${ }^{6} \mathrm{DIU}$ - dispositivo intrauterino. 
Tabela 3. Uso dos métodos contraceptivos pelas brasileiras em idade reprodutiva ( 18 a 49 anos) segundo suas variáveis sociodemográficas.

\begin{tabular}{|c|c|c|c|c|c|c|c|c|}
\hline \multirow{3}{*}{$\begin{array}{c}\text { Variáveis } \\
\text { Sociodemográficas }\end{array}$} & $\begin{array}{c}\text { Contraceptivo } \\
\text { oral } \\
\end{array}$ & Cirúrgicos $^{1}$ & Camisinhas $^{2}$ & $\begin{array}{c}\text { Dupla } \\
\text { proteção }^{3}\end{array}$ & $\begin{array}{c}\text { Outros } \\
\text { métodos }^{4}\end{array}$ & $\begin{array}{c}\text { Outros } \\
\text { hormonais }^{5}\end{array}$ & $\mathrm{DIU}^{6}$ & \multirow{3}{*}{$\mathrm{p}^{\star \star \star \star *}$} \\
\hline & $\%^{\star}[\mathrm{R}]^{\star \star *}$ & $\%^{\star}[\mathrm{R}]^{* * *}$ & $\%^{\star}[\mathrm{R}]^{\star * *}$ & $\%^{\star}[\mathbf{R}]^{\star * *}$ & $\%^{\star}[\mathrm{R}]^{\star * *}$ & $\%^{\star}[\mathbf{R}]^{\star * *}$ & $\% *[\mathrm{R}]^{\star * *}$ & \\
\hline & IC 95\% $\%^{\star *}$ & IC $95 \%{ }^{\star *}$ & IC $95 \%{ }^{\star *}$ & IC $95 \%{ }^{\star *}$ & IC $95 \%{ }^{* *}$ & IC $95 \%{ }^{\star *}$ & IC 95\%** & \\
\hline Região & & & & & & & & $<0,0001$ \\
\hline \multirow[t]{2}{*}{ Norte } & $16,5 \%[3]$ & $32,5 \%[1]$ & $23,6 \%[2]$ & $9,1 \%[5]$ & $10,0 \%[4]$ & $7,7 \%[6]$ & $0,6 \%[7]$ & \\
\hline & $14,4-18,8$ & $29,4-35,8$ & $20,9-26,6$ & $7,6-10,8$ & $7,8-12,7$ & $6,3-9,3$ & $0,4-1,1$ & \\
\hline \multirow[t]{2}{*}{ Nordeste } & $27,1 \%[2]$ & $35,7 \%[1]$ & $15,3 \%[3]$ & $6,9 \%[5]$ & $6,9 \%[6]$ & $7,4 \%[4]$ & $0,9 \%[7]$ & \\
\hline & $24,9-29,4$ & $33,2-38,2$ & $13,6-17,0$ & $5,9-8,1$ & $5,6-8,3$ & $6,2-8,7$ & $0,6-1,2$ & \\
\hline \multirow[t]{2}{*}{ Centro-Oeste } & $32,0 \%[2]$ & $34,4 \%[1]$ & $12,0 \%[3]$ & $8,9 \%[4]$ & $6,5 \%[5]$ & $5,1 \%[6]$ & $1,1 \%[7]$ & \\
\hline & $28,8-35,3$ & $31,4-37,5$ & $10,3-14,0$ & $7,3-10,8$ & $5,2-8,2$ & $4,0-6,5$ & $0,8-1,6$ & \\
\hline \multirow[t]{2}{*}{ Sudeste } & $38,6 \%[1]$ & $21,5 \%[2]$ & $12,9 \%[3]$ & $10,9 \%[4]$ & $7,9 \%[5]$ & $5,6 \%[6]$ & $2,6 \%[7]$ & \\
\hline & $36,2-41,1$ & $19,3-24,0$ & $11,2-14,7$ & $9,2-12,8$ & $6,6-9,4$ & $4,6-7,0$ & $1,9-3,5$ & \\
\hline \multirow[t]{2}{*}{ Sul } & $44,7 \%[1]$ & $13,4 \%[4]$ & $13,8 \%[2]$ & $13,6 \%[3]$ & $7,5 \%[5]$ & $4,5 \%[6]$ & $2,5 \%[7]$ & \\
\hline & $41,4-48,1$ & $11,3-15,8$ & $11,3-16,7$ & $11,4-16,1$ & $5,9-9,5$ & $3,2-6,2$ & $1,7-3,8$ & \\
\hline Zona de moradia & & & & & & & & $<0,0001$ \\
\hline \multirow[t]{2}{*}{ Urbana } & $34,0 \%[1]$ & $24,7 \%[2]$ & $14,9 \%[3]$ & $10,5 \%[4]$ & $7,7 \%[5]$ & $6,3 \%[6]$ & $2,0 \%[7]$ & \\
\hline & $32,6-35,5$ & $23,3-26,1$ & $13,8-16,0$ & $9,6-11,6$ & $6,9-8,5$ & $5,6-7,1$ & $1,6-2,4$ & \\
\hline \multirow[t]{2}{*}{ Rural } & $35,8 \%[1]$ & $34,4 \%[2]$ & $11,6 \%[3]$ & $5,8 \%[5]$ & $7,3 \%[4]$ & $4,1 \%[6]$ & $1,0 \%[7]$ & \\
\hline & $32,6-39,1$ & $31,2-37,8$ & $9,9-13,6$ & $4,5-7,4$ & $5,3-10,1$ & $2,9-5,7$ & $0,5-2,2$ & \\
\hline Estado Civil & & & & & & & & $<0,0001$ \\
\hline \multirow[t]{2}{*}{ Com companheiro } & $35,3 \%[1]$ & $32,6 \%[2]$ & $12,1 \%[3]$ & $5,2 \%[5]$ & $7,8 \%[4]$ & $4,4 \%[6]$ & $1,1 \%[7]$ & \\
\hline & $33,2-37,4$ & $30,5-34,7$ & $10,9-13,4$ & $4,3-6,2$ & $6,7-9,1$ & $3,6-5,4$ & $0,8-1,6$ & \\
\hline \multirow[t]{2}{*}{ Sem companheiro } & $33,4 \%[1]$ & $20,6 \%[2]$ & $16,3 \%[3]$ & $13,8 \%[4]$ & $7,5 \%[6]$ & $7,3 \%[5]$ & $1,1 \%[7]$ & \\
\hline & $31,6-35,3$ & $19,2-22,1$ & $15,0-17,8$ & $12,4-15,3$ & $6,5-8,5$ & $6,4-8,4$ & $0,8-1,6$ & \\
\hline Cor & & & & & & & & $<0,0001$ \\
\hline \multirow[t]{2}{*}{ Branca } & $39,0 \%[1]$ & $21,2 \%[2]$ & $13,6 \%[3]$ & $11,3 \%[4]$ & $7,9 \%[5]$ & $4,2 \%[6]$ & $2,9 \%[7]$ & \\
\hline & $36,7-41,2$ & $19,5-22,9$ & $12,2-15,3$ & $9,8-12,9$ & $6,8-9,2$ & $3,4-5,1$ & $2,2-3,8$ & \\
\hline \multirow[t]{2}{*}{ Preta/Parda } & $30,1 \%[2]$ & $30,2 \%[1]$ & $15,1 \%[3]$ & $8,9 \%[4]$ & $7,2 \%[6]$ & $7,6 \%[5]$ & $1,0 \%[7]$ & \\
\hline & $28,4-31,8$ & $28,4-31,9$ & $13,9-16,5$ & $7,9-9,9$ & $6,4-8,2$ & $6,6-8,7$ & $0,7-1,4$ & \\
\hline \multirow[t]{2}{*}{ Amarela/indígena } & $32,7 \%[1]$ & $23,3 \%[2]$ & $15,4 \%[3]$ & $7,2 \%[6]$ & $12,0 \%[4]$ & $8,8 \%[5]$ & $0,6 \%[7]$ & \\
\hline & $22,3-45,1$ & $15,8-33,1$ & $9,7-23,5$ & $4,3-11,9$ & $5,1-25,8$ & $4,9-15,1$ & $0,2-2,0$ & \\
\hline
\end{tabular}

nas utilizam pouco a dupla proteção, uma vez que esse método ficou na sexta posição em seu ranking.

As mulheres de 25 a 34 anos seguem o ranking nacional, e as de 35 a 49 anos utilizam quase duas vezes mais os métodos cirúrgicos do que o ACO. As mulheres mais jovens utilizam mais preservativos, uma vez que, quando somado o uso das camisinhas e da dupla proteção, a prevalência chega a quase $36 \%$, enquanto a das mulheres mais velhas é de $17,6 \%$. As mulheres de 18 a 24 anos apresentam apenas $0,3 \%$ de prevalência do DIU.

As mulheres que estudaram de 9 a 11 anos seguem o ranking nacional, já as mais escolarizadas utilizam mais o DIU do que as outras. Comparando-as com as de menor escolaridade, essa diferença é de seis vezes. As mulheres com menor nível de escolaridade utilizam mais as esterilizações $(39,7 \%)$ do que outros MC.

Ter ou não plano de saúde não modifica o ranking dos $\mathrm{MC}$, uma vez que, em ambos os casos, segue-se o padrão nacional. Porém, há diferenças importantes nas prevalências. Um exemplo disso seria o DIU, usado quase quatro vezes mais pelas que têm plano do que por aquelas que não têm.

\section{Discussão}

Os resultados encontrados evidenciaram que grande parte das brasileiras utiliza algum méto- 
Tabela 3. Uso dos métodos contraceptivos pelas brasileiras em idade reprodutiva (18 a 49 anos) segundo suas variáveis sociodemográficas.

\begin{tabular}{|c|c|c|c|c|c|c|c|c|}
\hline \multirow{3}{*}{$\begin{array}{c}\text { Variáveis } \\
\text { Sociodemográficas }\end{array}$} & $\begin{array}{c}\text { Contraceptivo } \\
\text { oral } \\
\end{array}$ & Cirúrgicos $^{1}$ & Camisinhas $^{2}$ & $\begin{array}{c}\text { Dupla } \\
\text { proteção }^{3}\end{array}$ & $\begin{array}{c}\text { Outros } \\
\text { métodos }^{4}\end{array}$ & $\begin{array}{c}\text { Outros } \\
\text { hormonais }^{5}\end{array}$ & DIU $^{6}$ & \multirow{3}{*}{$\mathrm{p}^{\star * * *}$} \\
\hline & $\%^{*}[\mathrm{R}]^{* * *}$ & $\%^{\star}[\mathrm{R}]^{\star * *}$ & $\%^{\star}[\mathrm{R}]^{\star * *}$ & $\%^{\star}[\mathrm{R}]^{* * *}$ & $\%^{\star}[\mathrm{R}]^{\star * *}$ & $\%^{\star}[\mathbf{R}]^{\star \star \star}$ & $\% *[R]^{\star * *}$ & \\
\hline & IC 95\% ${ }^{* *}$ & IC 95\%** & IC 95\%** & IC 95\% ${ }^{\star *}$ & IC $95 \%{ }^{* *}$ & IC 95\% ${ }^{\star *}$ & IC 95\% ${ }^{\star *}$ & \\
\hline Idade & & & & & & & & $<0,0001$ \\
\hline \multirow[t]{2}{*}{ 18-24 anos } & $43,5 \%[1]$ & $1,7 \%[6]$ & $17,4 \%[3]$ & $18,3 \%[2]$ & $8,3 \%[5]$ & $10,5 \%[4]$ & $0,3 \%[7]$ & \\
\hline & $39,8-47,2$ & $1,1-2,6$ & $15,0-20,2$ & $15,5-21,4$ & $6,8-10,1$ & $8,6-12,7$ & $0,2-0,6$ & \\
\hline \multirow[t]{2}{*}{ 25-34 anos } & $40,7 \%[1]$ & $18,1 \%[2]$ & $14,3 \%[3]$ & $10,9 \%[4]$ & $7,1 \%[5]$ & $7,2 \%[6]$ & $1,7 \%[7]$ & \\
\hline & $38,5-43,0$ & $16,4-19,8$ & $12,9-15,9$ & $9,7-12,4$ & $6,0-8,4$ & $6,1-8,4$ & $1,2-2,3$ & \\
\hline \multirow[t]{2}{*}{$35-49$ anos } & $24,0 \%[2]$ & $45,2 \%[1]$ & $12,8 \%[3]$ & $4,8 \%[5]$ & $7,6 \%[4]$ & $2,76 \%[6]$ & $2,77 \%[7]$ & \\
\hline & $22,1-26,1$ & $43,0-47,5$ & $11,5-14,3$ & $4,0-5,8$ & $6,6-8,8$ & $2,2-3,5$ & $2,1-3,7$ & \\
\hline Escolaridade & & & & & & & & $<0,0001$ \\
\hline \multirow[t]{2}{*}{$0-8$ anos } & $30,0 \%[2]$ & $39,7 \%[1]$ & $11,4 \%[3]$ & $5,8[5]$ & $6,6 \%[4]$ & $5,7 \%[6]$ & $0,8 \%[7]$ & \\
\hline & $27,9-32,3$ & $37,3-42,1$ & $10,2-12,8$ & $4,8-6,9$ & $5,6-7,9$ & $4,8-6,8$ & $0,5-1,2$ & \\
\hline \multirow[t]{2}{*}{ 9-11 anos } & $36,3 \%[1]$ & $19,5 \%[2]$ & $16,2 \%[3]$ & $11,7 \%[4]$ & $7,5 \%[5]$ & $7,2 \%[6]$ & $1,5 \%[7]$ & \\
\hline & $34,4-38,2$ & $18,0-21,2$ & $14,8-17,8$ & $10,4-13,1$ & $6,5-8,7$ & $6,2-8,3$ & $1,0-2,3$ & \\
\hline \multirow[t]{2}{*}{$12 \mathrm{ou}+$ anos } & $36,9 \%[1]$ & $16,0 \%[2]$ & $15,4 \%[3]$ & $13,6 \%[4]$ & $10,0 \%[5]$ & $3,1 \%[7]$ & $5,1 \%[6]$ & \\
\hline & $33,3-40,6$ & $13,6-18,6$ & $12,9-18,2$ & $10,8-16,8$ & $7,9-12,7$ & $2,1-4,5$ & $3,8-6,9$ & \\
\hline Plano de saúde & & & & & & & & $<0,0001$ \\
\hline \multirow[t]{2}{*}{ Sim } & $36,8 \%[1]$ & $20,8 \%[2]$ & $14,2 \%[3]$ & $11,5 \%[4]$ & $8,4 \%[5]$ & $4,6 \%[6]$ & $3,8 \%[7]$ & \\
\hline & $34,3-39,4$ & $18,6-23,2$ & $12,5-16,0$ & $9,8-13,5$ & $7,0-10,0$ & $3,6-5,9$ & $2,8-5,1$ & \\
\hline \multirow[t]{2}{*}{ Não } & $33,0 \%[1]$ & $28,2 \%[2]$ & $14,6 \%[3]$ & $9,2 \%[4]$ & $7,3 \%[5]$ & $6,7 \%[6]$ & $1,0 \%[7]$ & \\
\hline & $31,5-34,7$ & $26,7-29,8$ & $13,5-15,8$ & $8,3-10,2$ & $6,5-8,2$ & $5,9-7,6$ & $0,7-1,3$ & \\
\hline
\end{tabular}

Fonte: Elaborado pelos autores.

do de contracepção, e o motivo mais respondido pelas que não usam foi querer ou não se importar de engravidar. Atualmente, o contraceptivo hormonal oral se constitui como o método mais usado pelas mulheres, e o DIU, o menos utilizado, entre as categorias estudadas. Pode-se inferir ainda que o tipo de método utilizado pela mulher tem relação com sua condição socioeconômica.

Mesmo que grande proporção de mulheres use algum tipo de contracepção, mais da metade das gestações no Brasil não são planejadas e/ ou indesejadas ${ }^{3}$. Uma possível justificativa para essa incoerência seria que os métodos encontrados como mais utilizados são os de curta duração, ou seja, dependem da usuária para garantir sua efetividade, o que pode gerar falhas. Essa alta prevalência por métodos temporários com pouca durabilidade, como os contraceptivos hormonais orais, preservativos, diafragma, tabela e contraceptivo de emergência também foi evidenciada em estudo anterior que avaliou contracepção em países da América Latina ${ }^{6}$.

Foi identificado que a maioria das mulheres que não utiliza algum tipo de contracepção não tem indicação realmente, uma vez que elas não se importam ou querem ter uma gestação. Além disso, uma pequena parcela das brasileiras não usa algum $\mathrm{MC}$ porque não sabe aonde ir, a quem procurar para ter informações ou não sabe como utilizá-lo. Isso permite concluir que ainda existem falhas no planejamento reprodutivo em nosso país, pois não alcança todas as mulheres.

Existem lacunas a serem preenchidas nas políticas públicas, visto que as últimas estratégias programáticas que consideraram a saúde sexual e reprodutiva das mulheres brasileiras foram a Rede Cegonha, de 2011, e o Plano Nacional de Políticas para as Mulheres (PNPM), de 2013 a 2015. Outro fato importante foi que, em 2019, pela primeira vez o documento da Organização 
Mundial de Saúde (OMS), que firma compromissos acerca da saúde sexual e reprodutiva das populações junto aos demais países, não foi assinado pelo Brasil ${ }^{20}$, podendo ser considerado um marco dos vários retrocessos vivenciados mais recentemente nesse campo no país.

Pode-se considerar, ainda, que as melhorias obtidas no âmbito da saúde sexual e da saúde reprodutiva são decorrentes de políticas públicas estruturantes anteriores, como o Mais Saúde: Direito de Todos, que integrava o Programa de Aceleração do Crescimento (PAC) de 2007. Esse foi o responsável por expandir as ações do planejamento reprodutivo no país ${ }^{21}$ e pela inversão do primeiro com o segundo lugar no ranking nacional de MC comparado com os resultados da PNDS de $2006^{11}$. A Secretaria de Políticas para as Mulheres (SPM), de 2003, também tem importante papel nesses avanços. Em 2005, ela criou o PNPM, que tinha como principal objetivo reforçar a coordenação e articulação de políticas de promoção da igualdade de gênero. Em 2013, o PNPM foi revisitado e revisado (2013-2015), implementando em uma das suas linhas de ação a assistência ao planejamento reprodutivo dos brasileiros de forma integral, além de promover os direitos sexuais e reprodutivos das mulheres, de maneira intersetorial ${ }^{22}$. No entanto, em 2016 foram feitos drásticos cortes orçamentários na pasta de Saúde das Mulheres e, desde então, o PNPM não foi renovado ${ }^{23}$. Mais recentemente, por meio do Decreto no 9.417 , de 20 de junho de $2018^{24}$, a SPM foi transferida para o Ministério da Mulher, da Família e dos Direitos Humanos. Essas mudanças resultam na perda do espaço da saúde das mulheres nas políticas públicas brasileiras, o que pode gerar significativos impactos no PR que precisam ser constantemente monitorados no país.

Sobre os tipos de métodos de contracepção utilizados, os cirúrgicos, que antes lideravam o ranking ${ }^{11}$, perderam seu posto para os contraceptivos orais. A industrialização, a inserção das mulheres no mercado de trabalho, a elevação da escolaridade, a urbanização, o empoderamento feminino e o desejo de controlar o ciclo menstrual são algumas hipóteses para a maior adesão das mulheres aos $\mathrm{ACO}^{25}$. As pílulas são eficazes se tomadas corretamente, práticas e não interferem na vida sexual do casal ${ }^{26}$. Também são adquiridas facilmente no balcão das farmácias no Brasil além de estarem disponíveis gratuitamente no serviço público do Sistema Único de Saúde (SUS).

A utilização de hormônios aumentou no Brasil, pois a última pesquisa sobre PR mostrou que aproximadamente uma em cada cinco mulheres de 15 a 49 anos utilizavam pílula ${ }^{11}$. No presente, evidenciou-se que uma em cada três usam esse método e cerca de metade das brasileiras utiliza algum tipo de hormônio (oral, injetável ou local, como o implante) para evitar a gravidez. Por outro lado, como já exposto, esses métodos são em sua maioria de curta duração e dependem da utilização correta pela usuária ${ }^{27}$. Além disso, há diversas evidências de efeitos colaterais e alterações fisiológicas no corpo da mulher ocasionados pelo uso prolongado dos $\mathrm{ACO}^{26}$. No Brasil, por exemplo, cerca de $20 \%$ das mulheres que utilizavam ACO não deveriam fazê-lo, pois possuíam alguma contraindicação ${ }^{28}$. Tal fato reforça que o corpo feminino, até mesmo quando não é indicado, é muito medicalizado.

O declínio dos métodos cirúrgicos é um indicador importante, uma vez que nos mostra que hoje as mulheres estão tendo mais informações e opções de escolha para outros métodos também eficazes porém reversíveis. Acrescenta-se, ainda, que as mulheres mais vulneráveis são as mais esterilizadas, pois as que mais utilizam métodos cirúrgicos são as da região Norte, as que moram em zona rural, pretas ou pardas, com menor escolaridade e que não possuem plano de saúde.

Apenas uma a cada quatro brasileiras utiliza camisinha. Isso mostra que a maioria das mulheres com vida sexual ativa não utiliza proteção contra as IST em suas relações sexuais. Já é possível perceber os resultados das relações sexuais desprotegidas no cenário brasileiro. Em 2017, houve o maior número de novos casos diagnosticados de HIV dos últimos anos, totalizando $42.420^{29}$. A sífilis adquirida também aumentou drasticamente. Em 2010, a taxa da doença foi de $2 \%$, sete anos depois foi de $58,1 \%$, ou seja, um aumento de mais de $50 \%{ }^{30}$.

A dupla proteção é o método mais eficaz e efetivo contra a gravidez inesperada e as IST, uma vez que ele é a combinação de uso dos preservativos com algum MC moderno ${ }^{31}$. Os resultados mostram que a dupla proteção foi mais utilizada pelas mulheres da região Sul, da zona urbana, brancas, com maior nível de escolaridade e que têm plano de saúde. As que menos utilizam são as que vivem no Nordeste, na zona rural, pretas e pardas, com menor escolaridade e que não têm plano de saúde. Com isso, pode-se aferir que há diferenças de acesso e informação de acordo com o grupo socioeconômico e demográfico em que a mulher está inserida.

Por outro lado, as mulheres das regiões Norte e Nordeste são as que mais utilizam somente ca- 
misinha em suas relações. Mulheres pretas, pardas, amarelas e indígenas também utilizam mais esse método do que as brancas. Os preservativos não necessitam de prescrição, são facilmente encontrados no SUS e, se comparados aos outros métodos, são mais simples de utilizar ${ }^{31}$. Dessa forma, pode-se inferir que essas mulheres utilizam mais preservativos não porque é o único método que previne contra as IST ou porque o escolheram, mas porque é o mais acessível, uma vez que as regiões Norte e Nordeste tem menos acesso aos meios sociais, tendo assim maior índice de vulnerabilidade.

Os denominados "outros métodos" de contracepção abordados neste trabalho estão na quinta posição do ranking, com a prevalência de quase $8 \%$, superando os outros métodos hormonais e o DIU. Essa categoria é composta por métodos com pouca eficácia e combinações de dois ou mais métodos que não trazem algum tipo de benefício para a usuária, sendo essas combinações consideradas incoerentes ${ }^{31}$. Não por acaso, essa categoria é mais utilizada pelas mulheres que vivem em uma das regiões com menos acesso aos bens e serviços da sociedade do Brasil: a Norte. Tal fato reforça a necessidade de melhorias no serviço de PR dessas brasileiras, já que supomos que essas escolhas incoerentes são feitas pela ausência de informações ou por não confiarem em apenas um método. O enfermeiro é responsável em promover práticas educativas, aconselhamento e atividades clínicas que empoderem, por meio de informações, homens e mulheres na decisão de se reproduzirem ou não ${ }^{3}$, o que pode estar falho nos serviços.

Neste estudo, observamos que a região e a zona de moradia se relacionam com o MC utilizado pela mulher, assim como ter plano de saúde, cor da pele/raça e escolaridade. Essa relação se dá no sentido de que as brasileiras com algum grau de vulnerabilidade são duplamente prejudicadas. Ou seja, soma-se uma vulnerabilidade social indiretamente aferida pelas características sociodemográficas à maior vulnerabilidade em relação ao PR. Essas desigualdades em saúde podem ser exemplificadas pelo fato de as mulheres com baixa escolaridade apresentarem um padrão de contracepção mais próximo das mulheres que vivem em países de baixa renda, apesar do ranking de todas as mulheres ainda estar distante daquele encontrado em países ricos e desenvolvidos ${ }^{5,6,10,32}$.

Uma explicação para essa situação pode advir da limitação da capacidade de escolher entre diferentes alternativas, decorrentes da situação de vida vulnerável no contexto individual ${ }^{33}$, sendo a livre escolha informada um ponto-chave que deve sustentar o serviço de $\mathrm{PR}^{34}$. Sabe-se que o princípio de equidade do SUS tem como objetivo ofertar mais para aqueles que têm menos ${ }^{19}$. Com as iniquidades em saúde apontadas neste trabalho, pode-se afirmar que esse princípio precisa ser retomado de forma mais contundente ao ofertar PR às mulheres com vulnerabilidades sociais, uma vez que são as que mais necessitam.

Os métodos contraceptivos denominados LARC são aqueles que possuem longa duração e são reversíveis, além disso, sua eficácia não depende integralmente da usuária ${ }^{27}$. Os LARC são considerados altamente eficazes e econômicos, porém, mesmo com tantos benefícios, são mais utilizados pelas mulheres com maior renda e que possuem plano de saúde 6 , o que também foi constatado neste estudo.

O DIU é um exemplo de LARC, porém apenas duas em cada 100 brasileiras o utilizam como MC. Mesmo o SUS disponibilizando o DIU de cobre para as usuárias, possivelmente há empecilhos para a sua utilização. Nesse contexto, algumas hipóteses são: mitos sobre sua eficácia e funcionamento, critérios falsos de contraindicação, necessidade de profissionais especializados para sua inserção ${ }^{35}$, questões religiosas, dificuldade no acesso a exames e consultas para acompanhamento e a falta de informações sobre seus benefícios e sua ação.

A presente pesquisa apresenta algumas limitações, como as diversas combinações de dois ou mais métodos considerados incoerentes. Além da hipótese da falta de informação, podemos pensar que a pergunta do inquérito não limita para o MC utilizado no momento, o que pode gerar erros de compreensão. Outro fato foi a laqueadura e a vasectomia não terem sido incluídas no questionário como MC, mas sim como motivos para não evitar uma gestação. Além de ser equivocado, pois os métodos cirúrgicos são métodos de contracepção ${ }^{6,8}$, impede de relacioná-los ao uso dos preservativos, o que configuraria uma dupla proteção. Outra falha do questionário foi o fato de as lésbicas não poderem responder sobre o uso de preservativo em suas relações sexuais, contradizendo a Política Nacional de Saúde Integral de Lésbicas, Gays, Bissexuais, Travestis e Transexuais, de $2013^{36}$. A impossibilidade de avaliar as respostas às perguntas sobre os motivos de não utilizar MC e o tipo de MC utilizado, quando abertas, também foi uma fragilidade. Por último, não foi possível saber qual a composição do ACO e do injetável retratado, tampouco a classe do DIU. Essas limitações do questionário poderiam 
ser consideradas para se avançar nos próximos inquéritos nacionais que incluírem o tema do PR. Outra limitação foi não ter incluído mulheres de 15 a 17 anos, podendo haver uma superestimação do uso, pois geralmente usam pouco os $\mathrm{MC}^{37}$. Destaca-se que, apesar dessas limitações, foi possível desenvolver a pesquisa por meio da criação de categorias de MC mais amplas.

\section{Conclusões}

Observamos mudanças importantes no ranking nacional, sobretudo pela diminuição da prevalência do uso de métodos definitivos, apesar de ainda estar distante dos rankings em países de primeiro mundo, nos quais métodos de longa duração são mais utilizados com maior frequência. Os métodos utilizados pelas brasileiras estão relacionados diretamente a variáveis socioeconômicas e demográficas das mesmas. Além disso, não houve diminuição da prevalência do não uso de $\mathrm{MC}$ e existem desigualdades de acesso à contracepção no país. Sendo assim, o Brasil precisa investir mais em políticas públicas que ampliem o acesso e o conhecimento no campo da saúde sexual e reprodutiva para essas mulheres que mais necessitam. Também concluímos que é preciso expandir o alcance dos métodos LARC no SUS, por meio de mais informações sobre suas vantagens e seu funcionamento, bem como com a capacitação dos profissionais de saúde para incrementar a oferta do método.

\section{Colaboradores}

RE Trindade apoiou a concepção do estudo, conduziu a análise e interpretação dos dados, liderou a escrita da primeira versão, participou das revisões críticas e da preparação da versão final. BB Siqueira e TF Paula participaram da análise e interpretação dos dados, da escrita da primeira versão e das revisões críticas do artigo. MS Felisbino-Mendes realizou a concepção do estudo, o delineamento, supervisionou a análise e a interpretação dos dados e a escrita da primeira versão. Também participou da revisão crítica e contribuiu na edição e preparação da versão final. Todos os autores aprovaram a versão final do manuscrito.

\section{Agradecimentos}

TF Paula e BB Siqueira agradecem à Coordenação de Aperfeiçoamento de Pessoal de Nível Superior (CAPES) pela bolsa de mestrado demanda social.

\section{Financiamento}

Ministério da Saúde, nº 86, 2014. 


\section{Referências}

1. Instituto Brasileiro de Geografia e Estatística (IBGE). Brasil em síntese [página na Internet]. [acessado 2019 Abr 12]. Disponível em: https://brasilemsintese.ibge. gov.br/populacao/taxas-de-fecundidade-total.html.

2. Instituto Brasileiro de Geografia e Estatística (IBGE). Sinopse do Censo Demográfico 2012 [página na Internet]. [acessado 2019 Maio 20]. Disponível em: https:// censo2010.ibge.gov.br/sinopse/index.php?dados= $4 \& u f=00$.

3. Viellas EF, Domingues RMSM, Dias MAB, Gama SGN, Theme Filha MM, Costa JV, Bastos MH, Leal MC. Assistência pré-natal no Brasil. Cad Saude Publica 2014; 30(Supl. 1):S85-S100.

4. UNFPA - United Nations Population Fund. Fecundidade e dinâmica da população brasileira. Brasília: UNFPA; 2018.

5. New JR, Cahill N, Stover J, Gupta YP, Alkema L. Levels and trends in contraceptive prevalence, unmet need, and demand for family planning for 29 states and union territories in India: a modelling study using the Family Planning Estimation Tool. Lancet Glob Health 2017; 5(3):e350-e358.

6. De Leon RGP, Ewerling F, Serruya SJ, Silveira MF, Sanhueza A, Moazzam A, Becerra-Posada F, Coll CVN, Hellwig F, Victora CG, Barros AJD. Contraceptive use in Latin America and the Caribbean with a focus on long-acting reversible contraceptives: prevalence and inequalities in 23 countries. Lancet Glob Health 2019; 7(2):e227-e235.

7. Instituto de Brasileiro de Geografia e Estatística (IBGE). Censo Demográfico 2010: Banco Sidra [página na Internet]. [acessado 2019 Maio 20]. Disponível em: https://sidra.ibge.gov.br/tabela/3193\#resultado.

8. Ewerling F, Victora CG, Raj A, Coll CVN, Hellwig F, Barros AJD. Demand for family planning satisfied with modern methods among sexually active women in low-and middle-income countries: who is lagging behind? Reproductive Health 2018; 15(1):42-46.

9. Barros JVS, Wong LLR. Prevalência, conhecimento e tipos de métodos contraceptivos utilizados pelas mulheres, segundo o tipo de união: um estudo para Brasil e México. XVIII Encontro Nacional de Estudos Populacionais. São Paulo: ABEP; 2012.

10. Habyarimana F, Ramroop S. Spatial analysis of socio-economic and demographic factors associated with contraceptive use among women of childbearing age in Rwanda. Int J Environ Res Public Health 2018; 15(11):23-83.

11. Brasil. Ministério da Saúde (MS). Centro Brasileiro de Análise e Planejamento. Pesquisa Nacional de Demografia e Saúde da Criança e da Mulher - PNDS 2006 dimensões do processo reprodutivo e da saúde da criança. Brasília: MS; 2009.

12. Brasil. Ministério da Saúde(MS). Política Nacional de Humanização: HumanizaSUS [página na Internet]. 2013 [acesso 2017 Jul 28]. Disponível em: https://www. gov.br/saude/pt-br/acesso-a-informacao/acoes-eprogra-mas/humanizasus.

13. Secretaria de Vigilância em Saúde. Painel de mortalidade materna [página na Internet]. [acessado 2019 Maio 20]. Disponível em: http://svs.aids.gov.br/dantps/centrais-de-conteudos/paineis-de-monitoramento/mortalidade/materna/.
14. Diniz D, Medeiro M, Madeiro A. Pesquisa nacional de aborto 2016. Cien Saude Colet 2017; 22(2):653-660.

15. Damacena GN, Szwarcwald CL, Malta DC, Souza Júnior PRB, Vieira MLFP, Pereira CA, Morais Neto OL, Silva Júnior JB. O processo de desenvolvimento da Pesquisa Nacional de Saúde no Brasil, 2013. Epidemiol Serv Saude 2015; 24(2):197-206.

16. Szwarcwald CL, Malta DC, Pereira CA, Vieira MLFP, Conde WL, Souza Júnior PRB, Damacena GN, Azevedo LO, Silva GA, Theme Filha MM, Lopes CS, Romero DE, Almeida WS, Monteiro CS. Pesquisa Nacional de Saúde no Brasil: concepção e metodologia de aplicação. Cien Saude Colet 2014; 19(2):333-342.

17. Andrade SSA, Stopa SR, Brito AS, Chueri PS, Szwarcwald CL, Malta DC. Prevalência de hipertensão arterial autorreferida na população brasileira: análise da Pesquisa Nacional de Saúde, 2013. Epidemiol Serv Saude 2015; 24(2):297-304.

18. Brasil. Ministério da Saúde (MS). Secretaria de Atenção à Saúde. Departamento de Ações Programáticas Estratégicas. Manual dos comitês de mortalidade materna. Brasília: MS; 2009.

19. Brasil. Ministério da Saúde (MS). Secretaria de Atenção à Saúde. Departamento de Ações Programáticas Estratégicas. Direitos sexuais, direitos reprodutivos e métodos anticoncepcionais. Brasília: MS; 2009.

20. Family Planning 2020 (FP2020). Summary of Commitments. London; 2017.

21. Brasil. Ministério da Saúde (MS). Secretaria de Atenção à Saúde. Departamento de Atenção Básica. Direitos, saúde Sexual e saúde reprodutiva: marcos legais e políticos. In: Saúde sexual e saúde reprodutiva. Cadernos de Atenção Básica, n. 26. Brasília: MS; 2013.

22. Brasil. Ministério da Saúde (MS). Presidência da República. Secretaria de Políticas para as Mulheres. Plano Nacional de Políticas para as Mulheres. Brasília: Secretaria de Políticas para as Mulheres; 2013.

23. Gonçalves R, Abreu S. Do plano nacional de políticas para as mulheres ao "machistério" de Temer. Revista de Políticas Públicas 2019: 22(2):753-771.

24. Brasil. Decreto no 9.417, de 20 de junho de 2018. Transfere a Secretaria Nacional de Políticas para Mulheres e o Conselho Nacional dos Direitos da Mulher da Secretaria de Governo da Presidência da República para o Ministério dos Direitos Humanos. Diário Oficial da União 2018; 21 jun.

25. Nucci MF. Seria a pílula anticoncepcional uma droga de "estilo de vida"? Ensaio sobre o atual processo de medicalização da sexualidade. Sex Salud (Rio J) 2012; 10:124-139.

26. Almeida APF, Assis MM. Efeitos colaterais e alterações fisiológicas relacionadas ao uso contínuo de anticoncepcionais hormonais orais. Rev. Eletrôn. Atualiza Saúde 2017; 5(5):85-93.

27. Brandão ER. Métodos contraceptivos reversíveis de longa duração no Sistema Único de Saúde: o debate sobre a (in)disciplina da mulher. Cien Saude Colet 2019; 24(3):875-879.

28. Corrêa DAS, Felisbino-Mendes MS, Mendes MS, Malta DC, Velasquez-Melendez G. Fatores associados ao uso contraindicado de contraceptivos orais no Brasil. Rev Saude Publica. 2017; 51:1-10. 
29. Brasil. Ministério da Saúde (MS). Secretaria de Vigilância em Saúde. Boletim epidemiológico HIV/Aids. Brasília: MS; 2018.

30. Brasil. Ministério da Saúde (MS). Secretaria de Vigilância em Saúde. Boletim epidemiológico síflis. Brasília: MS; 2017.

31. World Health Organization (WHO). Departament of Reproductive Health and Research and Johns Hopkins Bloomberg School of Public Health, Center for Communication Programs (CCP), Knowledge for Health Project. Family planning: a global handbook for providers (2018 update). Baltimore and Geneva: CCP and WHO; 2018.

32. Beson P, Apipah R, Adomah-Afari A. Modern contraceptive use among reproductive-aged women in Ghana: prevalence, predictors, and policy implications. BMC Womens Health 2018; 18(1):157.

33. Comissão Nacional sobre Determinantes Sociais da Saúde. As causas sociais das iniquidades em saúde no Brasil. Rio de Janeiro: Fiocruz; 2008.

34. Carvalho MCMP, Paula CL, Queiroz ABA, Viana RB, Ferreira HC. Presença masculina no planejamento familiar: experiências e propostas de intervenções. $R e$ vista Enfermagem Atual 2018: 85(23):102-107.

35. Machado RB. Uso de dispositivos intrauterinos (DIU) em nulíparas. In: Federação das Associações Brasileiras de Ginecologia e Obstetrícia (FEBRASGO). Orientações e Recomendações FEBRASGO. São Paulo: FEBRASGO; 2018.

36. Brasil. Ministério da Saúde (MS). Secretaria de Gestão Estratégica e Participativa. Departamento de Apoio à Gestão Participativa. Política Nacional de Saúde Integral de Lésbicas, Gays, Bissexuais, Travestis e Transexuais. Brasília: MS; 2013.

37. Felisbino-Mendes MS, Paula TF, Machado IE, Oliveira-Campos M, Malta DC. Análise dos indicadores de saúde sexual e reprodutiva de adolescentes brasileiros, 2009, 2012 e 2015. Rev Bras Epidemiol 2018; 21(Supl. 1):e180013.

Artigo apresentado em 20/08/2019

Aprovado em 03/10/2019

Versão final apresentada em 05/10/2019

Editores-chefes: Romeu Gomes, Antônio Augusto Moura da Silva 